\section{Lichen sclerosus et atrophicans, scleroderma en coup de sabre and Lyme borreliosis}

\author{
Nicoletta Gubertini, Serena Bonin, \\ Giusto Trevisan \\ Department of Medical Sciences, Unit \\ of Dermatology, University of Trieste, \\ Trieste, Italy
}

\begin{abstract}
Lichen sclerosus et atrophicans (LSA) is a chronic, inflammatory skin disease of unknown etiology, characterized by atrophy.

We report a case of LSA with frontoparietal distribution, mimicking scleroderma en coup de sabre, causing scarring alopecia. The case was associated with Borrelia infection. The lesion improved with 2 cycles of antibiotic therapy with ceftriaxone $2 \mathrm{gr} /$ day i.v for 21 days associated with UVA-1 therapy and local and systemic vitamin E supply ( $400 \mathrm{mg} 2 \mathrm{x} /$ day per os for 3 months). This case stresses the importance of identifying clinical manifestations associated with Lyme disease and the use of tissue PCR to detect borrelial DNA in patients with these lesions, but characterized by negative serology for Borrelia.
\end{abstract}

\section{Introduction}

Lichen sclerosus et atrophicans (LSA) is a chronic inflammatory muco-cutaneous disorder, characterized by sclerotic and atrophic lesions, most commonly found in adult women. ${ }^{1}$

It affects the anogenital area in $85-98 \%$ of the cases and less frequently the extrgenital area. $^{2}$

The most commonly affected extragenital areas are the neck and the shoulders, ${ }^{1}$ but also the inner thighs, the submammary area, the wrist $^{2}$ and occasionally the oral mucosa. ${ }^{3}$

The involvement of the scalp is not frequent and its outcome could be similar to scarring alopecia, which could be the result of different diseases. ${ }^{4}$

The etiology of LSA is still unknown. Besides the genetic and local factors (Koebner phenomenon) and the autoimmune hypothesis, supported by the association of different autoimmune disorders, especially of thyroid origin ( $30 \%$ of cases), ${ }^{5,6}$ an infectious hypothesis has also been proposed. ${ }^{7,8}$

\section{Case Report}

A 57-year-old Caucasian woman presented with a history of asymptomatic frontoparietal lesion. Such lesion, which had been developing over the 3 previous years, was initially erythematous and became progressively atrophic and sclerodermic. The patient had been living in a highly endemic area for Borrelia burgdor feri, but she could not recall any tick bite or erythema chronicum migrans. She referred excellent health conditions. She also reported the simultaneous onset of migrating diffused myoarthralgias involving knees, hands, ankles, elbows, shoulders, as well as short-term memory impairment for two years, worsened previous seasonal insomnia for one year. Moreover, she presented migrating paresthesias involving the left side of the body for one month. The dermatological examination revealed an atrophic-sclerodermic lesion of about $10 \mathrm{~cm}$ in length and $3 \mathrm{~cm}$ in width, from the scalp to the centre of the forehead as reported in Figure 1. The skin was thin, inelastic, mother-of-pearl in shade, with erythematous margins of the lesion with subsequent scarring alopecia as well (Figure 1).

A skin biopsy was obtained from the scalp and after histological examination three different pathologists confirmed independently the diagnosis of LSA. It consisted of epidermal atrophy, oedema with superficial layer, hyperk eratosis and collagen production with cell rarefaction. The classical band of lymphocytes was present. Lymphoid infiltrate was observed even in the dermal-subepidermal junction indicating a possible later evolution of the LSA toward morphoea (Figure 2).

Serological IgM and IgG for Borrelia burgdorferi with enzyme-linked immunoassay test (ELISA) confirmed by Western Blot analysis was negative. Anti-nuclear antibodies (ANA), extractable nuclear antigen antibodies (ENA), anti-native DNA antibodies (n-DNA), anti-neutrophil cytoplasmic antibodies (ANCA) and erythrocyte sedimentation rate were negative.

PCR analysis for the detection of Borrelia burgdorferi was performed on DNA obtained from formalin-fixed paraffin-embedded skin biopsy, blood and urine as previously reported. ${ }^{9}$ PCR analysis was positive in all the specimens. After 6 months the PCR was repeated on DNA from blood and resulted negative.

The diagnosis of Lyme borreliosis was made on the basis of clinical data, supported by the PCR positivity for Borrelia genome.

The patient underwent antibiotic treatment with 2 cycles ceftriaxone $2 \mathrm{gr} /$ day i.v for 21 days. After about 6 months since the beginning of antibiotic therapy, the lesion had not progressed, all other clinical symptoms improved and blood PCR resulted negative. The patient
Correspondence: Serena Bonin, University of Trieste, Cattinara Hospital, Strada di Fiume 447, Surgical Pathology Blg, Lab Molecular Biology, 34149 Trieste, Italy. Tel. +39.040 .3996266 .

E-mail: sbonin@units.it

Key words: lichen sclerosus et atrophicans, frontoparietal distribution, Lyme borreliosis, PCR, Borrelia.

Received for publication: 20 July 2011. Accepted for publication: 31 August 2011.

This work is licensed under a Creative Commons Attribution NonCommercial 3.0 License (CC BYNC 3.0).

(C) Copyright S. Bonin et al., 2011

Licensee PAGEPress, Italy

Dermatology Reports 2011; 3:e27

doi:10.4081/dr.2011.e27

received vitamin $\mathrm{E} 400 \mathrm{mg} 2 \mathrm{x} /$ day per os for 3 months and applied topic vitamin E prior to UVA-1 therapy. UVA-1 treatment improved considerably her clinical condition. Thanks to this combined therapy a remarkable regression of the atrophic-sclerodermic lesion was observed.

\section{Discussion}

This report describes a case of LSA, which was unusual for the involvement of the scalp. The lesion was mimicking scleroderma en coup de sabre, which is a frontal or frontoparietal linear morphoea characterized by a linear band of depressed atrophy on skin and scalp.

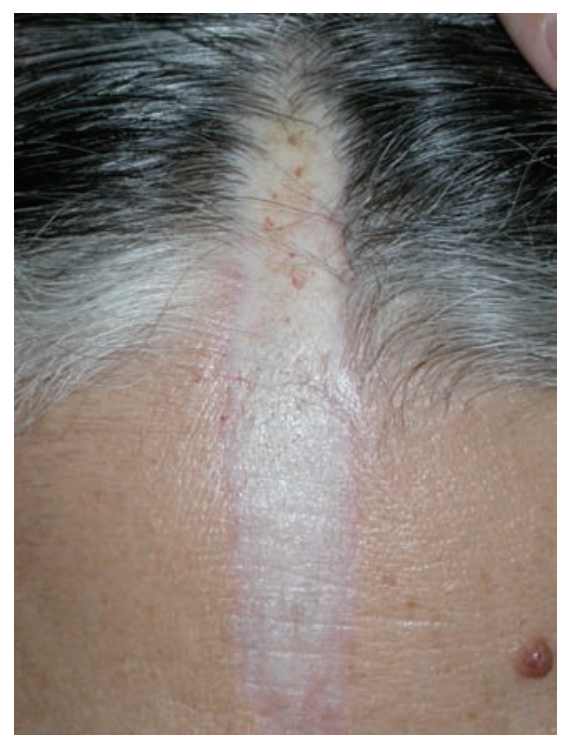

Figure 1. Lichen sclerosus et atrophicans with frontoparietal distribution, mimicking scleroderma en coup de sabre. 


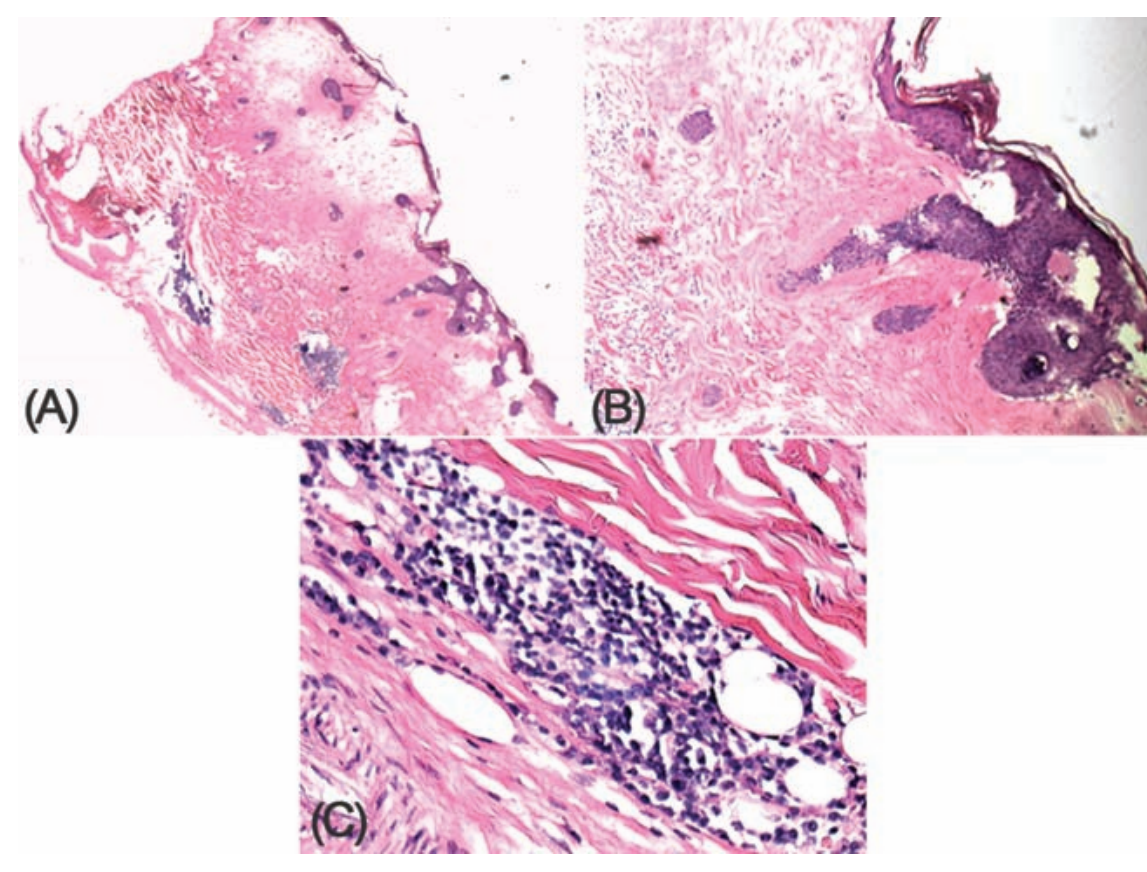

Figure 2. H\&E stained section of the lesion (A) 2,5 $\mathrm{x}$ magnification of the entire histological section, (B) 20x magnification of the lichen sclerosus et atrophicans features (C) $40 x$ magnification of the lymphatic infiltrate.

This lesion can frequently result in scarring alopecia.

The classical features of $\mathrm{LSA}^{1,10}$ are represented by hypopigmented papules that coalesce into white plaques with epidermal atrophy. Although anogenital LSA is associated with a risk of $4-5 \%$ of squamous cell carcinoma, extragenital lesions do not seem to carry any risk of malignant degeneration. ${ }^{2}$ The isolated linear frontoparietal involvement was described in few cases and may clinically simulate scleroderma en coup de sabre, requiring careful histopathological recognition. ${ }^{11}$ In this case, LSA has been diagnosed independently by three different expert pathologists. It has already been reported that overlap of histologic features between LSA and morphoea may occur, however in the reported case the clinical and the histologic features were not ambiguous of LSA. The possible later evolution toward morphoea in this case, due to the migration of the lymphatic infiltrate, is not unusual since morphoea and LSA may be closely related such that the latter could be considered the superficial expression of the same disease process which results in morphoea. ${ }^{12}$

Regarding the possibility of an infectious etiology of LSA, since the first proposal by Aberer and Stanek in $1987^{7}$ several European studies have debated on the association with Borrelia infection ${ }^{13}$ with conflicting results. Some atrophic skin diseases have been proposed as manifestation of Lyme borreliosis with contradictory results. ${ }^{8,14}$ The detection methods, the examined specimens, such as sera, skin biopsies and urine, together with the different geographic region could explain the conflicting results on the association of Borrelia with morphoea and lichen sclerosus et atrophicus. ${ }^{8}$ Moreover in the manifestation of long standing infection of Borrelia the paucity of microorganisms could lead to a low detection rate by PCR, especially when the analysis is performed on archival biopsies. ${ }^{8}$ No decision can be made to date as to whether $B b$ plays a role as causative agent of different types of circumscribed scleroderma and LSA. With regard to the disparate findings in different geographic areas, it can be speculated that LSA may be caused in some cases by $B b$ genotypes which are present in that area only. ${ }^{15} \mathrm{To}$ support this infectious etiology in endemic regions, several cases respond to antibiotic therapy for borreliosis, such as penicillin and ceftriaxone. ${ }^{16}$

The fact that in this case Borrelia DNA was detected both on DNA obtained from the biopsy and from blood and urine, strongly supports the hypothesis that Borrelia has a causative role on the onset of this unusual LSA. Moreover, the geographical location, Middle Europe, has been highly associated with Borrelia prevalence. ${ }^{8}$ Indeed, it is well known that there are significant geographic differences in Borrelia infections with a higher prevalence in areas in Middle Europe. ${ }^{8}$

To further support this theory, Lyme disease affects mostly the skin: about $80 \%$ of all Lyme borreliosis cases present skin manifestations. ${ }^{15}$ We recognise that classical dermato- logical events include erythema chronicum migrans (ECM), lymphadenosis benigna cutis (LABC) or borrellial lymphocytoma (BL) and acrodermatitis cronica et atroficans (ACA), but there is growing evidence that some cases of other cutaneous manifestations could be related to borrelial late infection, mostly Borrelia afzelii. ${ }^{13}$ The diagnosis of Lyme borreliosis is based on clinical data. Common laboratory tests are not usually revealing for the diagnosis of Lyme borreliosis and serologic tests support the diagnosis, but are not always essential in this regard. ${ }^{17}$

In this case of LSA mimicking scleroderma en coup de sabre, an associaton with Lyme borreliosis is proposed.

To our knowledge, this is the first case of LSA, mimicking scleroderma en coup de sabre, which was associated with Lyme borreliosis. Our findings indicate an association between this particular form of LSA and Borrelia, suggesting that Borrelia burgdorferi itself could represent a causative agent of this atypical form of LSA, however it cannot be excluded that Borrelia could be only one of the predisposing agent triggering it.

\section{Conclusions}

In conclusion we propose that tissue PCR for DNA of Borrelia should be performed in patients with LSA in endemic area, because it could represent a rare manifestation of Borreliosis, and in those cases LSA should be treated with proper antibiotic therapy in order to eradicate the microorganism.

\section{References}

1. Meffert JJ, Davis BM, Grimwood RE. Lichen sclerosus. Journal of the American Academy of Dermatology 1995;32:393-416; quiz 7-8.

2. Powell JJ, Wojnarowska F. Lichen sclerosus. Lancet 1999;353:1777-83.

3. Jimenez Y, Gavalda C, Carbonell E, et al. Lichen sclerosus of the oral mucosa: a case report. Med Oral Patol Oral Cir Bucal 2008;13:E403-6.

4. Chen W, Yang CC, Todorova A, et al. Hair loss in elderly women. Eur J Dermatol;20:145-51.

5. Birenbaum DL, Young RC. High prevalence of thyroid disease in patients with lichen sclerosus. J Reprod Med 2007;52:2830.

6. Powell J, Wojnarowska F, Winsey S, et al. Lichen sclerosus premenarche: autoimmunity and immunogenetics. Br J Dermatol 2000;142:481-4. 
7. Aberer E, Stanek G. Histological evidence for spirochetal origin of morphea and lichen sclerosus et atrophicans. Am J dermato Dermopathol 1987;9:374-9.

8. Zollinger T, Mertz KD, Schmid M, et al. Borrelia in granuloma annulare, morphea and lichen sclerosus: a PCR-based study and review of the literature. J Cutan Pathol 2010;37:571-7.

9. Pauluzzi P, Bonin S, Gonzalez Inchaurraga MA, et al. Detection of spirochaetal DNA simultaneously in skin biopsies, peripheral blood and urine from patients with erythema migrans. Acta Derm Venereol
2004;84:106-10.

10. Hallopeau H. Leçons cliniques sur le maladies cutanées et syphiliques. Union Med Can. 1887;43:472.

11. Coelho SW, Diniz LM, de Souza Filho JB. Lichen sclerosus et atrophicus - report of two cases with atipical presentations. An Bras Dermatol 2006;81 Suppl 3:S297-300.

12. Barnhill RL, ed. Textbook of Dermatopathology. 2nd ed: The McGraw-Hill Companies; 2004.

13. Eisendle K, Zelger B. The expanding spectrum of cutaneous borreliosis. G Ital Dermatol Venereol 2009;144:157-71.
14. Trevisan G. Atypical dermatological manifestations of Lyme borreliosis. Acta Dermatovenerol Alpina Pannonica Adriatica 2001;10:149-51.

15. Mullegger RR. Dermatological manifestations of Lyme borreliosis. Eur J Dermatol 2004;14:296-309.

16. Shelley WB, Shelley ED, Amurao CV. Treatment of lichen sclerosus with antibiotics. Int J Dermatol 2006;45:1104-6.

17. Bratton RL, Whiteside JW, Hovan MJ, et al. Diagnosis and treatment of Lyme disease. Mayo Clin Proc 2008;83:566-71. 